\title{
Pilot Study of Intra-Aortic Balloon Occlusion to Limit Morbidity in Patients with Adherent Placentation Undergoing Cesarean Hysterectomy
}

\author{
Elizabeth Blumenthal, MD, MBA ${ }^{1}$ Rashmi Rao, MD, MS ${ }^{2}$ Aisling Murphy, MD² \\ Jeffrey Gornbein, DrPH ${ }^{3}$ Richard Hong, MD ${ }^{4}$ John M. Moriarty, MD ${ }^{5}$ Daniel A. Kahn, MD, PhD \\ Carla Janzen, MD, $\mathrm{PhD}^{2}$
}

\footnotetext{
${ }^{1}$ Division of Maternal Fetal Medicine, Department of Obstetrics and Gynecology, University of California, Irvine, Orange County

2 Division of Maternal Fetal Medicine, Department of Obstetrics and Gynecology, University of California, Los Angeles, California

${ }^{3}$ Department of Biomathematics, University of California, Los Angeles, California

${ }^{4}$ Department of Anesthesia, University of California, Los Angeles, California

${ }^{5}$ Department of Interventional Radiology; University of California, Los Angeles, California
}

Am J Perinatol Rep 2018;8:e57-e63.
Address for correspondence Carla Janzen, MD, PhD, Division of Maternal Fetal Medicine, Department of Obstetrics and Gynecology, University of California, 10833 LeConte Avenue, Los Angeles, CA 90095 (e-mail: CJanzen@mednet.ucla.edu).

\begin{abstract}
Keywords

- placenta accreta

- intra-aortic balloon

- abnormal placentation

- placenta percreta

- cesarean hysterectomy

Objective We study whether using an intra-aortic balloon (IAB) during cesarean hysterectomy decreases delivery morbidity in patients with suspected morbidly adherent placentation.

Study Design This is a retrospective cohort study of deliveries complicated by suspected abnormal placentation between 2009 and 2016 comparing maternal and neonatal outcomes with an IAB placed prior to cesarean hysterectomy versus no IAB. The primary outcome included quantified blood loss (QBL).

Results Thirty-five cases were reviewed, 16 with IAB and 19 without IAB. No difference was seen in median QBL between the two groups (1,351 vs. $1,397 \mathrm{~mL}$; $p=0.90)$. There were no significant differences in overall surgical complications ( $19 \%$ $I A B, 21 \%$ no IAB; $p=0.86$ ), bladder complications (12 vs. $21 \% ; p=0.66$ ), intensive care unit admissions ( 12 vs. $26 \% ; p=0.41$ ), surgical duration ( 2.9 vs. 2.8 hour; $p=0.83$ ), or blood transfusions (median 2 vs. $2 ; p=0.27$ ) between the two groups. There was one groin hematoma at the balloon site that was managed conservatively. There were no complications involving thrombosis or limb ischemia in the IAB group. Conclusion While we did not detect statistically significant differences, larger studies may be warranted given the potential for extreme morbidity in these cases. This study highlights the potential use of an IAB in the management of these cases.
\end{abstract}

Invasive placentation is one of the most feared complications of pregnancy. Patients with morbidly adherent placentation -accreta, increta, and percreta-are at considerable risk of hemorrhage, disseminated intravascular coagulation, multi- ple blood product transfusions, acute respiratory distress syndrome, and damage to intra-abdominal organs during delivery and removal of the placenta. ${ }^{1}$ Reviews of the literature estimate that up to $25 \%$ of patients experience blood loss received

March 29, 2017

accepted after revision

February 23, 2018
DOI https://doi.org/

10.1055/s-0038-1641736. ISSN 2157-6998.
Copyright $\odot 2018$ by Thieme Medical Publishers, Inc., 333 Seventh Avenue, New York, NY 10001, USA. Tel: +1(212) 584-4662.
License terms

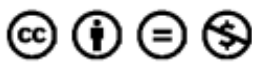


exceeding $5 \mathrm{~L}$; patients have a $40 \%$ chance of requiring more than 10 units packed red blood cells and an overall maternal mortality of $7 \% .^{2}$ The overall incidence of these cases has increased from $1 / 30,000$ deliveries in the 1960s to more recent reports suggesting an increase as high as $1 / 300$ over the past decade. ${ }^{3,4}$ Treatment for these disorders is generally cesarean hysterectomy, with rare cases of fertility-sparing measures ${ }^{5,6}$ for focally abnormal implantation. Recent studies have noted the while cesarean hysterectomies are increasingly concentrated in high-volume centers, this is not necessarily true across the country, particularly in rural areas with more limited referral capability. ${ }^{7}$ Given this, novel techniques that are relatively accessible and allow centers to optimize outcomes are needed.

Endovascular occlusive balloons have been employed to decrease regional perfusion pressure and limit the risk of hemorrhage during delivery in cases complicated by abnormal placentation. 8,9 Proponents of balloon techniques report that even with attempts at surgical ligation of uterine or internal iliac arteries, adequate control of bleeding occurs in less than $50 \%$ of cases. ${ }^{8,10}$, a However, in the gravid pelvis, there are rich anastomoses with significant collateral circulation through the cervical, ovarian, rectal, femoral, lumbar, and sacral arteries. These collateral communications contribute to the relative ineffectiveness of targeted vascular ligation. ${ }^{1,9}$ In a case-control study by Shrivastava et al comparing 19 patients who had intravascular (anterior iliac) balloon placement versus those who did not, there were no statistically significant differences seen in estimated blood loss, transfusion products, operative time, or postoperative hospital days. ${ }^{11}$ This study also noted the potential for harm; the complication rate directly attributed to the balloon was $15.8 \%$ including iliac artery and femoral artery thrombosis as well as a case of internal iliac artery dissection. In addition to these risks, the placement of iliac balloons requires the use of fluoroscopy, including access to equipment and appropriate personnel. This may require that the procedure be performed in a specific radiology suite if a $C$ arm cannot be brought into the operating room (OR). It also results in fetal fluoroscopic exposure.

As opposed to iliac balloons, intra-aortic balloons (IABs) may decrease the morbidity associated with cases of severely abnormal placentation given that more proximal occlusion may better address the problem of collateral circulation. Also, given that the aorta is a larger caliber vessel, it would be reasonable to believe that balloons placed here may not result in the harms found with iliac balloons. Finally, newer methods of placement of IABs under ultrasound guidance eliminate risks of fetal fluoroscopic exposure and enable placement in the same suite so that all procedures can be performed without moving the patient. It is also a procedure that can be performed by a larger number of different providers. For these reasons, our institution has piloted the use of prophylactic IAB occlusion in the management of cases with suspected placenta accreta. We hypothesize

\footnotetext{
a These studies investigated internal iliac ligation in patients with obstetric hemorrhage, in general, rather than only in patients with invasive placentation.
}

that the use of an IAB will decrease blood loss and other morbidity metrics in cases of abnormal placentation compared with usual care without balloons.

\section{Materials and Methods}

This was a retrospective cohort study of deliveries complicated by suspected abnormal placentation comparing cases where a prophylactic aortic balloon was placed to a control group managed with usual care (without the balloon). The control group was largely a historical control. In April 2013, the Departments of Obstetrics and Gynecology, Anesthesia and Interventional Radiology established a new accreta protocol at the University of California, Los Angeles (UCLA) which offered prophylactic aortic balloon placement. Therefore, to identify control cases, the UCLA database was searched using International Classification of Diseases, Ninth Revision, coding for "retained placenta" between 2009 and 2013. This code was selected to also account for cases with focal accreta, where conservative management may have been attempted. We performed a chart review of these cases to identify placenta accreta or more severe variants. After April 2013, we collected a prospective list of all accreta or more severe variants until April 2016. This study was approved by the Institutional Review Board at the UCLA (14-001688).

Inclusion criteria included cases identified as placenta accreta, increta, or percreta within the proposed timeframe. Exclusion criteria included cases where iliac balloons were used in addition to regular care, and gravid hysterectomies as termination procedures, if very limited information was available for the medical record and we could not reasonably identify reliable case information, ${ }^{\mathrm{b}}$ and if vaginal delivery was performed (and therefore we presumed that this was unlikely a case comparable to cases where an IAB was planned for use).

Maternal, neonatal, and operative baseline characteristics were abstracted from the electronic medical record. The primary outcome was quantified blood loss $(\mathrm{QBL})^{\mathrm{c}}$; several secondary outcomes including surgical complications, surgical duration, maternal intensive care unit (ICU) admission, ICU duration, postoperative complications, transfusions, postoperative hospital stay, postballoon complications, neonatal intensive care unit (NICU) admission, NICU stay duration, and Apgar scores were also collected. QBL and surgical duration were modeled using multivariable linear regression on the log scale to adjust for confounders. Covariates were chosen based on the biological plausibility that they could meaningfully impact results. Our sample size did not allow for extensive multivariate modeling or for using variable selection procedures from a larger pool of candidate predictors. QBL was adjusted for postdelivery diagnosis, number of prior cesarean deliveries, whether a fertility-sparing procedure was attempted, whether a delivery was unscheduled, if the

\footnotetext{
b UCLA adopted a new medical record system in June 2013, and selected records prior to this time were scanned into the system. c UCLA instituted a quantified blood loss program in 2009 to replace estimated blood loss. Given the time period of this study, this should have affected both control and case groups.
} 
pregnancy was a multiple gestation pregnancy, and whether general anesthesia was used.

Surgical duration was adjusted for the number of prior abdominal surgeries (cesarean and myomectomy), postdelivery diagnosis (percreta vs. no percreta), presence of preprocedure ureteral stents, and whether general anesthesia was administered prior to cesarean. QBL and surgical duration were adjusted on the log scale since their log values follow a normal (Gaussian) distribution. Other secondary outcomes were compared with univariate analysis. Binary variables were compared with Fisher's exact test. The nonparametric Wilcoxon rank-sum test was used to compare continuous variables. A sample size calculation was not initially performed as this is a pilot study based on a convenience sample of cases conducted thus far compared with a control group with standard care largely prior to implementation of the IAB.

\section{Results}

We identified 40 cases of abnormal placentation from the medical record between 2009 and 2016. Of these, we excluded five cases. - Fig. 1 outlines case inclusion and exclusion based on prespecified parameters. The majority $(14 / 19)$ of non-IAB cases were historic controls prior to the protocol change in April 2013. Five additional non-IAB cases were included in analysis occurring from May 2013 to March 2014. We believed the balloon decision was largely due to provider preference and ongoing familiarization with the balloon rather than inherent differences in the cases. No cases of placenta accreta or variants were excluded from analysis between April 2013 and April 2016.
Since April 2013, the IAB has been used in the management of 16 cases of abnormal placentation. We found no significant differences in baseline maternal or neonatal characteristics between the IAB and no-IAB groups (-Table 1). We found similar baseline operative characteristics; however, there was a significant difference in whether a fertility-sparing procedure was initially attempted. A fertility-sparing procedure was identified in chart review if the operative report described an attempt to remove the placenta. In 10 out of the 19 (52.6\%) no-IAB cases, a fertilitysparing procedure was initially attempted prior to performing cesarean hysterectomy, whereas this only occurred in 2 out of $16(12.5 \%)$ of the IAB cases. Additionally, more preprocedural ureteral stents were used in the IAB group versus the no-IAB group (56.2 vs. $10.5 \% ; p=0.01$ ).

Fluoroscopy was used for aortic balloon placement in 7/16 (44\%) of the patients in the IAB group. After 2015, all balloons were placed with intravascular ultrasound guidance, which did not require fluoroscopy (56\% of the total IAB cases). Those in the IAB group who received fluoroscopy exposure, the median duration was 0.8 minutes (range: $0.2-2.2$ minutes). The balloon was inflated at least once in 15/16 (94\%) cases. The median number of separate balloon inflations was 1 (range: 1-4). The median time of total balloon inflation was 60 minutes (range: 3-156 minutes). The maximum time of uninterrupted balloon inflation was 86 minutes (recorded in 14 out of 16 cases); the median time of uninterrupted balloon inflation was 48 minutes (-Fig. 2). The only balloon-related complication was one groin hematoma at the balloon site, which was managed conservatively. There were no recorded incidences of limb ischemia or thrombosis. Notably, heparin was not used during placement of the aortic balloons.

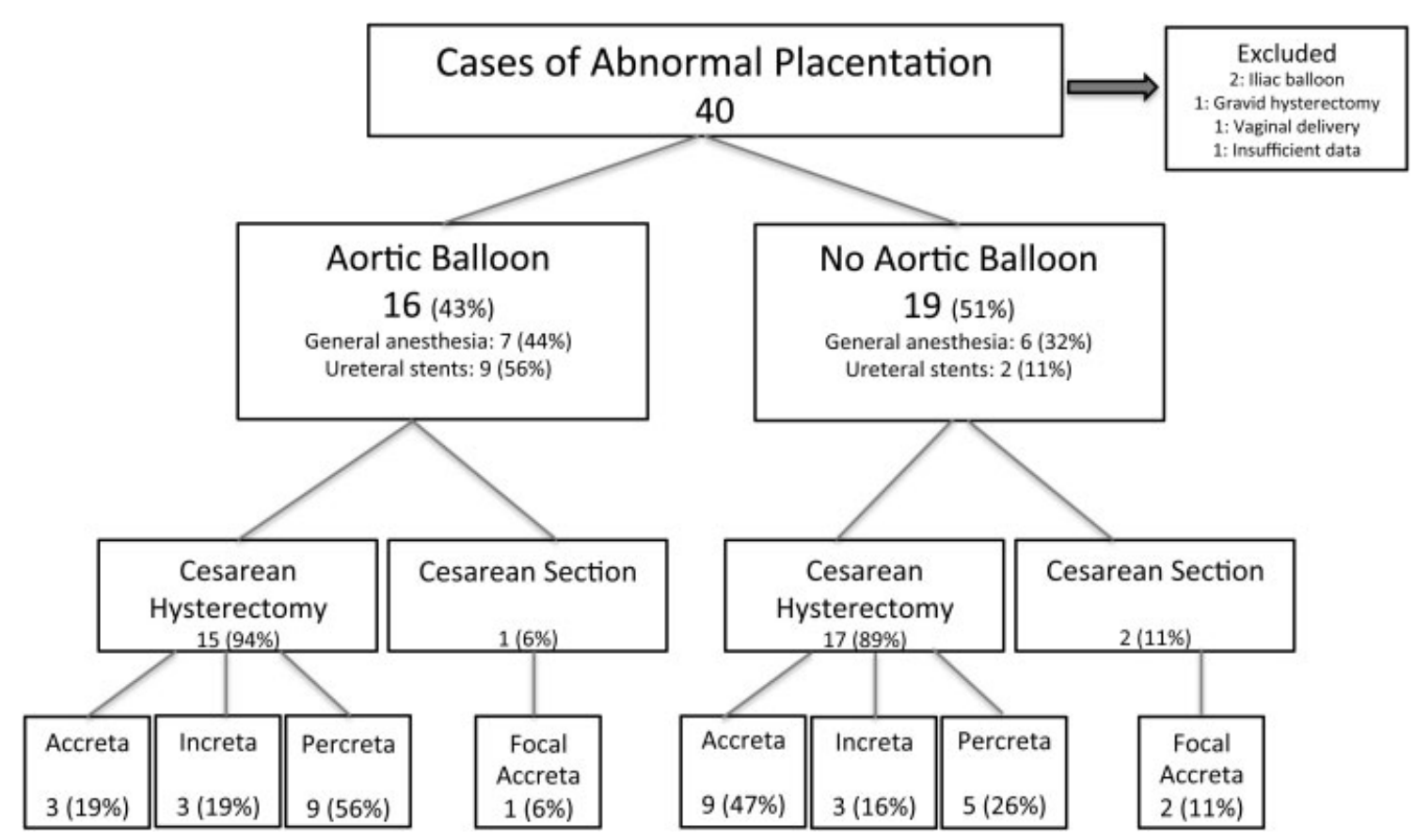

Fig. 1 Flow diagram representing case and control cohort selection. 
Table 1 Baseline maternal and neonatal characteristics

\begin{tabular}{|c|c|c|c|}
\hline & $\begin{array}{l}\text { Aortic balloon } \\
(N=16)\end{array}$ & $\begin{array}{l}\text { No balloon } \\
(N=19)\end{array}$ & $p$-Value \\
\hline \multicolumn{4}{|l|}{ Maternal characteristics } \\
\hline Age (years) & $35.5(25-41)$ & $34(27-40)$ & 0.75 \\
\hline Parity & $3(1-5)$ & $2(1-6)$ & 0.52 \\
\hline BMI $\left(\mathrm{kg} / \mathrm{m}^{2}\right)$ at time of delivery & $27(16-38)$ & $25(15-42)$ & 0.37 \\
\hline Number prior dilation and curettage & $0(0-2)$ & $0(0-3)$ & 0.95 \\
\hline Number prior cesarean deliveries & $2(0-5)$ & $1(0-6)$ & 0.59 \\
\hline Number prior myomectomy & $0(0)$ & $0(0-1)$ & 0.35 \\
\hline Number prior endometrial ablation & $0(0)$ & $0(0)$ & NA \\
\hline Multiple gestations & $2(12.5)$ & $2(10.5)$ & 0.85 \\
\hline Hypertension & $2(12.5)$ & $0(0)$ & 0.20 \\
\hline Diabetes (including gestational) & $0(0)$ & $1(5.3)$ & 0.35 \\
\hline Preeclampsia & $0(0)$ & $0(0)$ & NA \\
\hline \multicolumn{4}{|l|}{ Operative characteristics } \\
\hline Fertility-sparing procedure attempted & $2(12.5)$ & $10(52.6)$ & 0.03 \\
\hline Unscheduled delivery & $4(25)$ & $7(36.8)$ & 0.46 \\
\hline General anesthesia before cesarean & $4(26.7)$ & $4(21)$ & 0.70 \\
\hline General anesthesia during case & $7(43.8)$ & $6(31.6)$ & 0.50 \\
\hline Preprocedure ureteral stents placed & $9(56.2)$ & $2(10.5)$ & 0.01 \\
\hline \multicolumn{4}{|l|}{ Pathological characteristics } \\
\hline Percreta & $8(50)$ & $5(26)$ & 0.54 \\
\hline Increta & $3(18.8)$ & $3(15.8)$ & \\
\hline Accreta & $4(25)$ & $8(42.1)$ & \\
\hline Focal accreta & $1(6.25)$ & $2(10.5)$ & \\
\hline \multicolumn{4}{|l|}{ Neonatal characteristics } \\
\hline Betamethasone administered $48 \mathrm{~h}$ prior to delivery & $14(87.5)$ & $12(63.2)$ & 0.14 \\
\hline Female & $10(62.5)$ & $12(63.1)$ & 0.97 \\
\hline Gestational age at delivery & $34.4(30.3-36)$ & $35(29-41)$ & 0.29 \\
\hline Neonatal weight & $2,153(1,505-3,845)$ & $2,403(1,280-3,755)$ & 0.28 \\
\hline
\end{tabular}

Abbreviations: BMI, body mass index; NA, not available.

Note: Data are presented as median (range) or $n(\%)$.

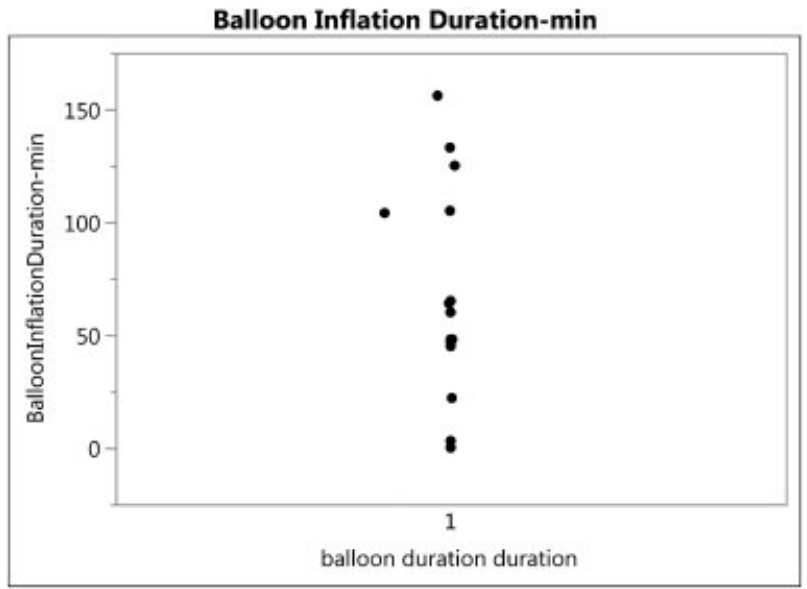

Fig. 2 jitter plot of overall time of aortic balloon inflation used in the 16 cases.
Median QBL was not different between the two groups after multivariable adjustment (1,351 vs. $1,397 \mathrm{~mL} ; p=0.90)$ (-Table 2). While balloon placement was not predictive of $\mathrm{QBL}$, we found that in cases where a fertility-sparing procedure was attempted, median blood loss was 1.9 times higher compared with when it was not attempted, controlling for the other six factors (QBL ratio: $1.93 ; p=0.05$ ).

We initially found a trend toward longer surgical duration in the IAB group ( 3.25 vs. 2.65 hours; $p=0.20$ ); however, after multivariable adjustment, adjusted median duration was almost equal between groups ( 2.90 vs. 2.81 hours; $p=0.83$ ) (-Table 2). As anticipated, cases of confirmed percreta (yes or no) were associated with longer surgical duration (median ratio: $1.41 ; p=0.002$ ). Interestingly, receiving general anesthesia prior to cesarean was associated with longer surgery (median ratio: $1.40 ; p=0.03$ ). Preprocedure ureteral 
Table 2 Primary and secondary outcomes

\begin{tabular}{|c|c|c|c|}
\hline & Aortic balloon $(N=16)$ & No balloon $(N=19)$ & $p$-Value \\
\hline \multicolumn{4}{|l|}{ Primary outcomes } \\
\hline Quantified blood loss (mL) & $2,007(900-20,000)$ & $2,112(500-8,664)$ & 0.83 \\
\hline Quantified blood loss $(\mathrm{mL})^{\mathrm{a}}$ & 1,351 & 1,397 & 0.90 \\
\hline \multicolumn{4}{|l|}{ Secondary outcomes } \\
\hline Surgical duration (hours) & $3.18(1.52-8.33)$ & $2.73(1.47-7.02)$ & 0.20 \\
\hline Surgical duration (hours) ${ }^{\mathrm{b}}$ & 2.90 & 2.81 & 0.83 \\
\hline Surgical complications, $n(\%)$ & $3(18.8)$ & $4(21.1)$ & 0.87 \\
\hline Vascular & $1(6.3)$ & $0(0)$ & 0.46 \\
\hline Bladder & $2(12.5)$ & $4(21)$ & 0.67 \\
\hline Ureter & $2(12.5)$ & $2(10.5)$ & 0.85 \\
\hline Gastrointestinal & $0(0)$ & $0(0)$ & NA \\
\hline Maternal ICU admission, $n$ (\%) & $2(12.5)$ & $5(26.3)$ & 0.42 \\
\hline ICU duration (days) & $0(0-5)$ & $0(0-2)$ & 0.43 \\
\hline Postoperative complications, $n(\%)$ & $3(18.8)$ & $4(25)$ & 0.67 \\
\hline Venous thromboembolism & $0(0)$ & $0(0)$ & NA \\
\hline Infection & $3(18.8)$ & $1(6.3)$ & 0.60 \\
\hline Ileus & $1(6.3)$ & $1(12.5)$ & 0.54 \\
\hline pRBC transfusions, $n(\%)$ & $2(0-23)$ & $2(0-15)$ & 0.27 \\
\hline Postballoon complications, $n(\%)$ & $1(6.3)$ & NA & NA \\
\hline Postoperative hospital stay (days) & $4(3-10)$ & $5(3-15)$ & 0.43 \\
\hline NICU admission, $n(\%)$ & $16(100)$ & $15(93.8)$ & 0.31 \\
\hline NICU stay duration (days) & $10(1-44)$ & $10(0-47)$ & 0.78 \\
\hline 1-min Apgar & $7(1-9)$ & $8(1-9)$ & 0.66 \\
\hline 5-min Apgar & $9(6-9)$ & $9(7-9)$ & 0.66 \\
\hline
\end{tabular}

Abbreviation: ICU, intensive care unit; NICU, neonatal intensive care unit; pRBC, packed red blood cell. Note: Data are presented as median (range) and $n(\%)$.

${ }^{a}$ Adjusted for postdelivery diagnosis, number prior cesarean, fertility-sparing procedure, unscheduled delivery, multiple gestations, and use of general anesthesia.

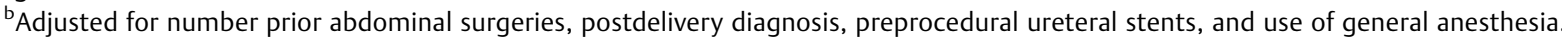

stent placement (yes or no) did not significantly affect surgical time (median ratio: $1.06 ; p=0.71$ ).

There were no significant differences in overall surgical complications between the two groups ( 3 out of 16 cases in the IAB had a complication vs. 4 out of 19 cases in the noballoon group; $p=0.87$ ). We were unable to run a multivariate analysis regarding surgical complications given the limitation of study size. There were no significant differences between the groups in terms of postoperative ICU admissions, median units of packed red blood cell transfusions, or postoperative hospital stay. We found similar neonatal outcomes between the two groups.

\section{Discussion}

This study did not find differences in QBL, surgical complications, or surgical duration when an IAB was used versus cases where the IAB was not used. The power of this trial to detect significant differences in the outcomes was limited by being a pilot study. There was an approximately $5 \%$ difference in $\mathrm{QBL}$ between the IAB group and the no-IAB group, and more than 3,000 patients would be needed in each arm to detect this difference. There were more pronounced (and more clinically relevant) differences suggested by the data in terms of ICU admissions (12.5\% in the IAB group vs. $26.3 \%$ in the non-IAB group; $p=0.42$ ), and future studies aimed at this difference might be both more feasible and warranted. In addition, IAB as an adjunct procedure for the most severe and complicated cases of adherent placentation may show more benefit than examining use in all cases. In our study, complications of the IAB itself were limited and minor. While this study was also not powered to prove safety, the preliminary data make it reasonable to consider larger studies. This study also describes the potential for ultrasound-guided placement and information regarding balloon inflation duration.

Aortic balloon occlusion has been described as an emergency measure in the treatment of penetrating abdominal trauma and aortoenteric fistula. ${ }^{12}$ It also has been described 
as a temporizing measure in cesarean hysterectomy for placenta percreta at the time of postpartum hemorrhage. ${ }^{9,13}$ Our institution investigated its use after other studies described the ease of the procedure and the impression of improved surgical visualization with bleeding control. ${ }^{9}$

The procedure is relatively available. While performed by Interventional Radiology at our institution, other providers familiar with aortic endovascular procedures could perform this procedure at other sites. This procedure is more accessible than balloon placement in smaller caliber vessels and may provide more downstream benefit than previously seen in balloon studies with smaller caliber vessels. The use of intravascular ultrasound-guided placement also opens up the possibility of all procedures being performed in one OR suite without the necessity of a C-arm or fetal fluoroscopic exposure.

Potential risks of aortic balloon inflation may include aortic rupture, potential for distal plaque embolization, inability to deflate the balloon, and inability to withdraw the large caliber compliant balloon through the sheath. ${ }^{14}$ Experience with aortic cross-clamping in procedures such as abdominal aortic aneurysm repair reports that occlusion for 45 to 60 minutes is generally safe, with continuous extremity pulse oximetry. ${ }^{9,15}$ Our study demonstrated very few complications and none related to limb ischemia or thrombosis. Additionally, inflation times occasionally exceeded the 60-minute threshold, and it is likely that a younger, healthier pregnant population can tolerate longer periods of inflation than older individuals with prior vascular disease who have previously been included in these studies. While we had only a minor complication, groin site hematoma, it is noteworthy that Wei et al had more clinically significant complications such as arterial thrombosis and femoral nerve ischemia. ${ }^{16}$ They reported on monitoring for limb ischemia throughout the procedure; however, it is less clear how long the balloon was inflated in their series. In our series, the balloon was inflated up to a maximum of 156 minutes in total, however only to 86 minutes in a sustained fashion. The majority of cases were inflated for 60 minutes or less. While younger, healthier patients may be able to sustain longer inflation times than cardiovascular patients, this is still a notable risk, and caution regarding the duration of balloon inflation is important. Our sample size is insufficient to demonstrate safety, and these parameters should be closely watched in a center performing these procedures.

Our study provides one of the largest series of IAB cases used for the prophylactic control of bleeding currently published, specifically with the most severe class of invasive placentation where this type of procedure is most likely to be helpful. Wei et al recently published a similar study comparing the use of an aortic balloon versus no balloon in 45 patients. However, a minority of their patients $(n=3)$ had the most severe of the placental variants, placenta percreta. ${ }^{16}$ Our series includes 13 patients with placenta percreta; 8/16 (50\%) of the cases in the IAB arm had this most morbid variant. In addition, they describe an attempt to remove the placenta in all of their cases, where this attempt at fertility sparing was only performed in $2 / 16$ of our IAB cases where a focal accreta was suspected. An attempt to remove the placenta would be expected to increase potential for blood loss (as our data demonstrate). In addition, all of the cases published by Wei et al involved placement of the balloon under fluoroscopy, whereas the majority of our cases included ultrasound-guided placement.

Limitations of this study include its retrospective nature. We were unable to fully control for all relevant confounding variables. Additionally, we had limited ability to evaluate differences in outcome metrics or safety given our small sample size; however, this report is intended to serve as a pilot for use of a new technology. In a condition with increasing incidence and such severe maternal morbidity including blood loss as well as surgical complications, new technologies that may enhance care are relevant to other centers, and pilot studies are important.

Ultimately, a larger analysis is needed to fully evaluate if there could be benefit in terms of QBL, surgical complications, transfusions, or ICU admissions for patients in addition to evaluating potential harms. The difference potentially suggested by these data in terms of ICU admission could be clinically relevant, and powering a future study to investigate this outcome may be most reasonable. In addition, invasive placentation is not homogeneous, and the most severe variants including placenta percreta may be the situations where this technology could be most beneficial. We believe that our study demonstrates the feasibility of using an IAB as a prophylactic tool and lays the groundwork for larger studies.

\section{References}

1 Chestnut DH. The Fred Hehre Lecture 2006. Lessons learned from obstetric anesthesia. Int J Obstet Anesth 2008;17(02):137-145

2 Miller DA, Chollet JA, Goodwin TM. Clinical risk factors for placenta previa-placenta accreta. Am J Obstet Gynecol 1997; 177(01):210-214

3 Belfort MA. Indicated preterm birth for placenta accreta. Semin Perinatol 2011;35(05):252-256

4 Silver RM. Abnormal placentation: placenta previa, vasa previa, and placenta accreta. Obstet Gynecol 2015;126(03):654-668

5 Sentilhes L, Gromez A, Clavier E, Resch B, Verspyck E, Marpeau L. Fertility and pregnancy following pelvic arterial embolisation for postpartum haemorrhage. BJOG 2010;117(01):84-93

6 Balayla J, Bondarenko HD. Placenta accreta and the risk of adverse maternal and neonatal outcomes. J Perinat Med 2013;41(02): 141-149

7 Govindappagari S, Wright JD, Ananth CV, Huang Y, D'Alton ME, Friedman AM. Risk of peripartum hysterectomy and center hysterectomy and delivery volume. Obstet Gynecol 2016;128(06): 1215-1224

8 Kidney DD, Nguyen AM, Ahdoot D, Bickmore D, Deutsch LS, Majors C. Prophylactic perioperative hypogastric artery balloon occlusion in abnormal placentation. AJR Am J Roentgenol 2001; 176(06):1521-1524

9 Masamoto H, Uehara H, Gibo M, Okubo E, Sakumoto K, Aoki Y. Elective use of aortic balloon occlusion in cesarean hysterectomy for placenta previa percreta. Gynecol Obstet Invest 2009;67(02): 92-95

10 Clark SL, Phelan JP, Yeh SY, Bruce SR, Paul RH. Hypogastric artery ligation for obstetric hemorrhage. Obstet Gynecol 1985;66(03): 353-356 
11 Shrivastava V, Nageotte M, Major C, Haydon M, Wing D. Casecontrol comparison of cesarean hysterectomy with and without prophylactic placement of intravascular balloon catheters for placenta accreta. Am J Obstet Gynecol 2007;197(04):402.e1-402.e5

12 Harma M, Harma M, Kunt AS, Andac MH, Demir N. Balloon occlusion of the descending aorta in the treatment of severe post-partum haemorrhage. Aust N Z J Obstet Gynaecol 2004;44(02):170-171

13 Usman N, Noblet J, Low D, Thangaratinam S. Intra-aortic balloon occlusion without fluoroscopy for severe postpartum haemorrhage secondary to placenta percreta. Int J Obstet Anesth 2014;23(01):91-93
14 Søvik E, Stokkeland P, Storm BS, Asheim P, Bolås O. The use of aortic occlusion balloon catheter without fluoroscopy for lifethreatening post-partum haemorrhage. Acta Anaesthesiol Scand 2012;56(03):388-393

15 Irahara T, Sato N, Moroe Y, Fukuda R, Iwai Y, Unemoto K. Retrospective study of the effectiveness of Intra-Aortic Balloon Occlusion (IABO) for traumatic haemorrhagic shock. World J Emerg Surg 2015;10(01):1

16 Wei X, Zhang J, Chu Q et al. Prophylactic abdominal aorta balloon occlusion during caesarean section: a retrospective case series. Int J Obstet Anesth 2016;27:3-8 